Urologe 2019.58:692-696

https://doi.org/10.1007/s00120-019-0922-5

Online publiziert: 9. April 2019

(c) Springer Medizin Verlag GmbH, ein Teil von Springer Nature 2019

\section{Derzeitige Position und Werdegang}

Nach dem Studium der Medizin in Würzburg habe ich meine Ausbildung zur Fachärztin für Urologie an der Klinik für Kinderurologie der Universität Regensburg begonnen und anschlieBend an den urologischen Kliniken der Kliniken Nordoberpfalz und der Universität Regensburg fortgesetzt. Als Fachärztin wurde ich Ende 2011 durch einen Headhunter für eine Position in der Pharmaindustrie angeworben; nach mehreren Interviews habe ich mich für dieses Angebot entschieden, nicht ahnend, ob dieser Weg der richtige für mich ist und was ein Job in der Industrie eigentlich genau bedeutet. Nach 2 Jahren als Medical Advisor im Fachbereich Uroonkologie Deutschland für die Firma Janssen (Johnson and Johnson) folgten 2 Jahre im Bereich EMEA (Europe, Middle East, Africa) als Regional Therapeutic Area Expert. Hier war es meine Aufgabe, klinische Studien unter Berücksichtigung der Patientenpopulation, des medizinischen Bedarfs, gesundheitsökonomischer und weiterer Anforderungen effizient und strategisch zu planen. Seit 2016 arbeite ich als Direktorin im Bereich Research and Development Global, wo ich u. a. medizinisch verantwortlich bin für uroonkologische Phase-III-Studien. Meine akademische Forschung und Lehre habe ich an der LMU München fortgesetzt. Daneben arbeite ich flexibel in einer Privatpraxis in Freising und mittels Videosprechstunde,

Sabine Brookman-May

Regensburg, Deutschland

\title{
Medizinischer und digitaler Fortschritt im Jahr 2025
}

\section{Medizinische Qualität, Patientenfokus und gesundheits-ökonomische Aspekte sinnvoll in Einklang bringen}

da ich die Tätigkeit direkt am Patienten nicht komplett aufgeben wollte. Außerdem bin ich Mutter von drei Kindern im Alter von einem bis 18 Jahren und Geschäftsführerin eines Start-ups für digitale medizinische Lösungen.

\section{Was macht an der aktuellen Position Freude, was belastet? Wo bestehen Probleme? Würde man alles wieder so machen?}

Meine Tätigkeit macht insbesondere in der Kombination Freude - klinische Forschung in der Industrie gepaart mit ärztlicher Tätigkeit am Patienten, akademischer Forschung und Lehre und der Entwicklung digitaler medizinischer Lösungen. Meine Tätigkeit in der Industrie ist abwechslungsreich, insbesondere durch den sich oft wechselnden Fokus in der klinischen Forschung und täglich neu auftretende Herausforderungen. Es macht mir Freude, an der Entwicklung onkologischer Medikamente mitzuwirken, mit denen wir unsere Patienten effizient behandeln können. Die internationale Tätigkeit, die sich für mich auf die Forschung in 26 Ländern weltweit erstreckt, bietet die Möglichkeit, mit vielen Ärzten unterschiedlicher Disziplinen zusammenzuarbeiten und mit ärztlichen Experten auf verschiedenen Gebieten bei der Entwicklung von Studienprotokollen sowie der wissenschaftlichen Publikation der Daten zu kooperieren. In großen pharmazeutischen Unternehmen ist es außerdem wichtig, auch innerhalb der Firma Netzwerke zu knüpfen, strategi- sche Partnerschaften zu entwickeln und interdisziplinär zusammenzuarbeiten (z.B. mit Bereichen wie Medical Affairs, Marketing, Sales, Health Economy und Reimbursement, Safety, Regulatory und anderen): das ermöglicht neben dem Blick über den medizinischen Tellerrand eine Erweiterung der eigenen Expertise auf angrenzenden und fachfremden Gebieten. Letztendlich ist es aber v.a. die Möglichkeit, Therapien für einen bestehenden medizinischen Bedarf $\mathrm{zu}$ entwickeln, die die ärztliche Tätigkeit in der Forschung sinnvoll macht.

Eine Tätigkeit in der Industrie beinhaltet einige Herausforderungen: gefordert ist in der Regel ein hohes Maß an Flexibilität, die Fähigkeit, sich neue Gebiete schnell und selbstständig $\mathrm{zu}$ erschließen und in wechselnde Tätigkeitsfelder einzuarbeiten. Es gibt hierarchische Strukturen, die sich von denen der akademischen und nichtakademischen ärztlichen Tätigkeit unterscheiden. Das bietet Vorteile und Nachteile - Titel zählen wenig bis nichts (außer evtl. in der Kommunikation mit externen Partnern), relevant ist das Ergebnis sowie die Qualität als Führungsperson, was auch Grundlage einer variablen (Bonus-)Vergütung ist. Strategische Netzwerkbildung über verschiedene Sektoren und das Verständnis, dass neben dem medizinischen Aspekt auch weitere zählen (u.a. Zulassungsoptionen und -beschränkungen, Entwicklungsoptionen, Anforderungen von Gesundheitsbehörden etc.) bzw. sich die medizinischen Anforderungen (auch an klinische Studien) von Land $\mathrm{zu}$ 
Land unterscheiden können, sind enorm wichtig. Weltweit gestalten sich nicht alle Aspekte der Medizin wie in Deutschland. Oft sind daher Kompromisse und pragmatische Lösungen notwendig, um klinische Forschung voranbringen $\mathrm{zu}$ können.

Beruflich würde ich alles wieder so machen - vorausgesetzt, ich würde wieder Medizin studieren (mittlerweile könnte ich mir bei der Vielzahl der verfügbaren Möglichkeiten evtl. auch andere Optionen vorstellen). Die Entscheidung gegen eine klassische akademische Karriere in Deutschland war eine bewusste Entscheidung. Die Struktur der Klinikhierarchien, die oft ineffiziente Art und Weise der akademisch-wissenschaftlichen Zusammenarbeit, bei der jeder den Fokus auf seine eigene Karriere legt, und die teilweise (natürlich von Klinik zu Klinik unterschiedliche) bestehende Inflexibilität hinsichtlich Neuerungen und Neustrukturierungen in der Medizin würden es mir nach wie vor erschweren, mich im Klinikalltag dauerhaft wohl zu fühlen. Andererseits gibt es aber auch in der Pharmaindustrie Situationen, die frustrieren können und wenig zielführend scheinen oder sind. Zudem wäre ich sicher auch nicht in jeder industriellen Position gut aufgehoben; in der klinischen Forschung habe ich jedoch eine sehr sinnvolle Tätigkeit gefunden. Im Gegensatz $\mathrm{zu}$ den meisten meiner ärztlichen Kollegen in der Industrie habe ich aber die ärztliche Betreuung von Patienten beibehalten - darauf möchte ich auch weiterhin nicht verzichten. Die kürzlich getroffene Entscheidung, auch noch mit der Entwicklung medizinischer Softwarelösungen $\mathrm{zu}$ beginnen, fußte dann v.a. darauf, dass in großen industriellen Unternehmen (selbst wenn diese sehr innovativ sind) manche Ideen dann doch etwas Zeit bis zur Finalisierung benötigen und ich meine Pläne zumindest innerhalb meiner primären Tätigkeit nicht realisieren konnte.

\section{Risiken für meine Tätigkeit und den Fachbereich Urologie}

Für einen industriell tätigen Arzt mit hoher Expertise und Erfahrungen in verschiedenen Sektoren der Pharmaindus- trie sowie einer vorherigen (oder zeitgleichen) akademischen Laufbahn gibt es zumindest keine Risiken hinsichtlich eines potentiellen Mangels an möglichen Arbeitsstellen. Ärzte, die bereits industrielle Erfahrung mit sich bringen, können sich in der Regel vor Angeboten anderer Firmen kaum retten. Den individuell richtigen Arbeitsplatz zu finden, erfordert dennoch in vielen Fällen eine wechselnde Tätigkeit in verschiedenen Bereichen und ggf. Firmen, da sich eine große Variabilität in den industriellen Tätigkeiten findet. Die forschende Pharmaindustrie wird weiterhin in gleichem bzw. wachsendem Umfang an neuen Medikamenten zur onkologischen Therapie (und zunehmend auch zur Prävention) forschen. Auch in anderen therapeutischen Bereichen ist eine Zunahme der Forschung zu verzeichnen (Immuntherapie, neurologische Forschung, Schmerztherapie). Ebenso wird im Bereich der antiinfektiösen Therapie entgegen oft gelesener Aussagen umfangreich geforscht, auch wenn oft vermutet wird, dass aufgrund des geringen Gewinns pro Einzelverordnung wenig Interesse an einer weiteren Forschung auf diesem Gebiet besteht. Dies unterstellt jedoch, dass die Pharmaindustrie ausschließlich gewinnorientiert forscht. Natürlich muss, wie in jeder Arztpraxis und Klinik, die wirtschaftliche Bilanz stimmen, allerdings werden Gewinne aus umsatzstärkeren Bereichen auch zur Kofinanzierung anderer Forschungsbereiche genutzt. Zudem führen eher günstige Medikamente (z. B. Hypertonusmedikation) gesundheitsökonomisch oft zu größeren Kosten als z.B. onkologische Medikamente, da sie einer deutlich größeren Zahl von Patienten über einen längeren Zeitraum verordnet werden.

Für das Fach Urologie sehe ich generell keine größeren Risiken, einerseits aufgrund des breiten Spektrums an urologischen Erkrankungen, andererseits aufgrund der demographischen Entwicklung, die zu einer weiteren Zunahme urologischer und uroonkologischer Krankheitsbilder führen wird. Ein geringes Risiko besteht ggf. darin, dass wir Urologen uns möglichen (technischen) Neuerungen, u. a. auch im Bereich Digitalmedizin und eHealth, nicht aufgeschlossen genug zeigen. Insgesamt ist die ärztliche urologische Gemeinschaft jedoch eher fortschriftlich eingestellt. Digitalisierung bedeutet aber nicht nur, das Smartphone als Informationsquelle zu nutzen und Patientenkarteien digital führen. Die digitale (R)evolution sollte aktiv von uns Ärzten gestaltet werden, bevor externe und nichtärztliche Anbieter das Feld der Digitalmedizin aufrollen. Weiterhin wird sich die Medizin insgesamt und auch die Urologie wahrscheinlich dahingehend entwickeln, dass weniger chirurgisch therapiert und bei manchen Diagnosen vermehrt medikamentös behandelt werden wird. Primär- und Sekundärprävention werden zunehmend wichtig und sinnvollerweise vermehrt in den Fokus rücken. Durch die demographische Entwicklung werden wir zunehmend multimorbide Patienten betreuen. Dafür wird weitere Expertise u.a. im geriatrischen Assessment und der Therapieplanung notwendig sein, die wir entweder selbst erwerben oder aber (strategisch evtl. eher nicht sinnvoll) anderen Fachbereichen überlassen können. Auch werden wir uns fragen müssen, welche Aufgaben anderen Fachgebieten überlassen werden sollten und welche nicht - Allgemeinmedizin (z. B. PSA-Screening, Vorsorge allgemein), Onkologie (medikamentöse Therapie, Palliativmedizin) und Radiologie (onkologische Diagnostik und Therapie) stehen hier in Konkurrenz.

Besteht ein Risiko auch dahingehend, dass Ärzte (teilweise) ersetzt oder ärztliche Tätigkeiten in andere Bereiche integriert werden können? Ich denke, dass dieses Risiko tatsächlich gegeben ist. Zum einen lassen sich manche ärztliche (oder vermeintlich ärztliche) Tätigkeiten auch durch ärztliche Assistenz erledigen. Relevanter ist jedoch die Frage, inwiefern wir generell ersetzbar sind, ggf. auch durch Algorithmen unter Nutzung von Künstlicher Intelligenz (KI) und Maschinenlernen. Schauen wir uns die aktuelle Trefferquote von Dr. Watson (IBM) und anderen KI-Systemen an, können wir davon ausgehen, dass in Zukunft die Diagnostik für bestimmte Krankheitsbilder ganz oder teilweise durch solche Systeme übernommen werden wird. Erste Krankenversicherer beginnen (verständlicherweise) bereits mit deren Integra- 
tion in den diagnostischen Ablauf. Data Science sollte aber nicht als Gefahr für den ärztlichen Beruf gesehen werden, sondern als Chance, die Medizin zu verbessern und effizienter zu gestalten. Die ärztliche Kunst wird dennoch weiter als solche gesehen werden und Bestand haben, denn nur ein Teil der ärztlichen Tätigkeit wird komplett zu ersetzen sein. Zudem ist maschinelles Lernen eine Weiterentwicklung ärztlichen Wissens - ganz ohne Ärzte geht es (zumindest bisher) noch nicht. Fachgebiete wie Radiologie oder Pathologie haben vermutlich ein größeres Risiko (aber damit auch größere Chancen), durch Nutzung von KI deutlich verändert zu werden, da Befundungen effektiv automatisiert und anhand lernender Algorithmen verbessert werden können.

Neben der Weiterentwicklung der Digitalisierung, die, egal, ob das von uns Ärzten begrüßt wird oder nicht, genutzt werden wird, werden auch weiterhin Wirtschaftlichkeit und Effizienz in der Medizin gefordert werden. Zunehmend werden neben den öffentlichen Gesundheitsträgern und den beteiligten Akteuren weitere Unternehmen mit vorrangig ökonomischer Zielsetzung in die Gesundheitsversorgung drängen. Auch pharmazeutische Unternehmen werden sich als Player in der allgemeinen Gesundheitsversorgung $\mathrm{zu}$ etablieren versuchen.

\section{Vision 2025}

Das Fach Urologie wird sich bis zum Jahr 2025 sicher deutlich verändern - in einigen Bereichen weniger, in anderen mehr. Insbesondere der technische Fortschritt (Industrie 4.0) wird auch vor der Urologie nicht Halt machen und damit Chancen bieten, aber auch Risiken in sich tragen. Meine Vision der Urologie im Jahr 2025 beinhaltet eine intelligente Nutzung der neuen Techniken und Kommunikationsmöglichkeiten, die so gestaltet wird, dass sie den ärztlichen Beruf verbessern, den Patientennutzen vergrößern und die $\mathrm{Zu}$ friedenheit von uns Ärzten in unserem Beruf steigern. Die Anwendung von Data Science, maschinellem Lernen und KI begrüße ich, da sie bei richtiger Nutzung die Möglichkeit bieten, die Gesundheits- versorgung insgesamt zu verbessern. Unsere ärztliche Mission muss sein, dass wir die Nutzung neuer Systeme selbst gestalten und diese sinnvoll weiterentwickeln. Die virtuelle Fernbehandlung wird es ermöglichen, Patienten auch über räumliche Distanzen adäquat betreuen und den Arztberuf damit auch flexibler gestalten zu können. Home Office und eine weitere ärztliche Tätigkeit z. B. während Elternoder Teilzeit für den klinisch tätigen Arzt werden damit teilweise möglich. Meine Vision beinhaltet auch, dass wir (ähnlich wie Piloten) virtuelle Realität und Simulation nutzen, um das operative Training zu verbessern. Damit werden in Zukunft nicht nur die operativ durch ihre Vorgesetzten begünstigten Assistenzärzte die Möglichkeit haben, strukturiert das chirurgische Handwerk zu erlernen, sondern ein effizientes operatives Training für alle möglich sein.

Auch die Pharmaindustrie wird sich wandeln - neben der Therapie von Krankheiten wird der Fokus zunehmend auf dem Bereich der Prävention und Disease Interception liegen. Data Science und KI werden genutzt werden, um klinische Studien effizienter zu gestalten und z. B. weniger Patienten in Kontrollarme einschließen zu müssen, da diese aus anderen Studien generiert werden können, ohne die statistische Power hinsichtlich der zu erreichenden Endpunkte zu gefährden.

Letztendlich hoffe ich, dass für uns Urologen auch im Jahr 2025 das Patientenwohl primärer Fokus und primäre Zielsetzung unseres Berufs darstellt, egal ob wir in Klinik, Praxis, Pharmaindustrie oder anderen Bereichen tätig sind. Dafür ist es notwendig, dass wir uns weder von anderen Fachbereichen (noch die einzelnen medizinischen Sektoren untereinander) abschotten, sondern die intersektorale und interdisziplinäre Zusammenarbeit verbessern. Auch die Fachverbände (allen voran DGU und BDU) müssen an einem Strang ziehen. Die Sicherung des eigenen Fachgebiets bzw. der eigenen urologischen Tätigkeit aus wirtschaftlichen Gründen und um des Status' Willen ist falsch motiviert, wenn dabei nicht das Patientenwohl im Vordergrund steht.

\section{Fazit}

Die Urologie im Jahr 2025 wird eine bessere sein. Jeder Fortschritt birgt Risiken, jedoch deutlich mehr Chancen in sich, vorausgesetzt wir integrieren unser ärztliches Wissen sinnvoll und nutzen technische Neuerungen zur Verbesserung der ärztlichen Tätigkeit. Die Entwicklung einer technologisch fortschrittlichen Urologie sollten wir nicht anderen Akteuren im Gesundheitswesen ohne ausreichende ärztliche Kompetenz überlassen. Vielmehr müssen wir eigene Konzepte entwickeln bzw. diese mitentwickeln, um die beste Qualität neuer Gesundheitslösungen zu ermöglichen.

Ich hoffe, dass wir weiterhin permanent den Status quo hinterfragen, um unseren Fachbereich effektiv weiterzuentwickeln. Obwohl ökonomische Überlegungen auch in der Medizin relevant sind, sollten finanzielle Aspekte und der Status des eigenen Fachbereichs nie Haupttreiber für Veränderungen sein, sondern immer das Patientenwohl und die Verbesserung der Medizin im Vordergrund stehen. Wirtschaftlichkeit und ärztliche Tätigkeit stehen dabei nicht im Widerspruch - was medizinisch sinnvoll und effektiv ist, kann meist auch mit entsprechender Wirtschaftlichkeit durchgeführt werden.

Letztendlich möchte ich noch einen Wunsch in mein Zukunftsszenario integrieren. Ich wünsche mir, dass Ärztinnen und Ärzte die Möglichkeit bekommen, sich auch ohne langfristigen Einfluss auf ihre Karriere auf Familie und Kinder einzulassen. Insbesondere wünsche ich mir, dass junge Ärztinnen im Jahr 2025 Männer an ihrer Seite haben, die mehr als die mittlerweile nahezu obligaten 2 Monate Elternzeit in Anspruch nehmen und ihnen zuhause den Rücken freihalten, während sie selbst arbeiten dürfen. Unser gemeinsames Ziel sollte sein, dass Sätze wie „Entweder ist man Mutter oder Chirurgin, für beides ist in der Chirurgie keine Zeit und kein Platz" (Prof. Izbicki, UKE Hamburg, Deutscher Ärztetag 2018) im Jahr 2025 weder in der Chirurgie noch in der Urologie formuliert werden, sondern Familie und Beruf effektiv vereinbart werden können. Wir werden nicht 
umhinkommen, die Urologie so zu gestalten, dass dies möglich sein wird.

\section{Korrespondenzadresse}

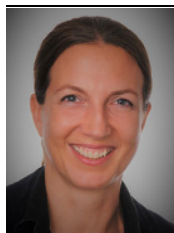

Prof. Dr. med.

Sabine Brookman-May

Regensburg, Deutschland

sabine.brookman-

may@email.de

Prof. Dr. med. Sabine Brookman-May Fachärztin für Urologie, FEBU, Medikamentöse Tumortherapie, Sportmedizin; Medizinische Direktorin, Global Research and Development, Uro-Onkologie, Janssen Pharma, USA

Interessenkonflikt. S. Brookman-May arbeitet als Medical Director, Global Reseach and Development für Janssen Pharma Research and Development (Los Angeles, CA, USA). Es besteht kein Interessenkonflikt hinsichtlich des vorliegenden Artikels.

\section{Lesen Sie Der Urologe online auf SpringerMedizin.de}

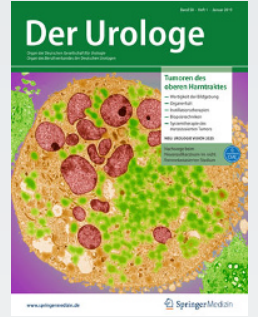

Auf SpringerMedizin.de erhalten Sie Zugang zu allen elektronisch verfügbaren Ausgaben und dem CME-Angebot Ihrer Zeitschrift - unabhängig davon, seit wann Sie Der Urologe abonniert haben. Außerdem können Sie die Zeitschrift mit dem E-Paper auch bequem auf Ihrem Tablet lesen.

So einfach erhalten Sie Zugang zum Online Archiv Registrieren Sie sich einmalig auf www.springermedizin.de/register

Geben Sie dabei Ihre Einheitliche Fortbildungsnummer (EFN) an.

- Ihr Benutzername entspricht Ihrer E-Mail-Adresse, Ihr Passwort können Sie frei wählen und später jederzeit unter "Mein Profil“ ändern.

- Falls Sie bereits ein (Print-) Abonnement bei uns haben, geben Sie bei der Registrierung die Lieferadresse Ihrer Zeitschrift an. Damit wird Ihr Abo-Zugang auf springermedizin.de freigeschaltet.
Sind Sie bereits bei SpringerMedizin.de registriert? Dann wird Ihr Zeitschriftenabonnement automatisch Ihrem Online-Nutzerkonto hinzugefügt. Sollten die Angaben Ihres Online-Accounts nicht eindeutig mit den Angaben Ihres Zeitschriften-Abonnements übereinstimmen, kann die Zuordnung nicht sicher erfolgen. In diesem Fall und bei allen anderen Fragen zum Online-Zugang kontaktieren Sie bitte unseren Kundenservice unter: Kundenservice@springermedizin.de

Telefonisch erreichen Sie die Hotline montags bis freitags von 9.00 bis 17.00 Uhr kostenfrei unter 0800-77 80777 sowie gebührenpflichtig aus dem Ausland unter $\hookleftarrow+4930884293600$ 
Hier steht eine Anzeige.

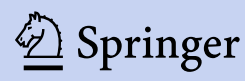

